\title{
Understanding patient outcomes after acute respiratory distress syndrome: identifying subtypes of physical, cognitive and mental health outcomes
}

\author{
Samuel M Brown, ${ }^{1,2,3}$ Emily L Wilson, ${ }^{1,2}$ Angela P Presson, ${ }^{4}$ Victor D Dinglas, ${ }^{5,6}$ \\ Tom Greene, ${ }^{4}$ Ramona 0 Hopkins, ${ }^{1,2,7}$ Dale M Needham, ${ }^{5,6,8}$ with the National \\ Institutes of Health NHLBI ARDS Network
}

\begin{abstract}
- Additional material is published online only. To view please visit the journal online (http://dx.doi.org/10.1136/ thoraxjnl-2017-210337).

For numbered affiliations see end of article.
\end{abstract}

\section{Correspondence to} Dr Samuel M Brown, Shock Trauma Intensive Care Unit, 5121 South Cottonwood Street, Murray, UT 84107, USA; samuel. brown@imail.org

Received 31 March 2017 Revised 23 June 2017

Accepted 10 July 2017 Published Online First 7 August 2017

\section{Linked}

- http://dx.doi.org/10.1136/ thoraxjnl-2017-210775

CrossMark

To cite: Brown SM,

Wilson EL, Presson AP, et al.

Thorax 2017:72:1094-1103.

\section{ABSTRACT}

Purpose With improving short-term mortality in acute respiratory distress syndrome (ARDS), understanding survivors' posthospitalisation outcomes is increasingly important. However, little is known regarding associations among physical, cognitive and mental health outcomes. Identification of outcome subtypes may advance understanding of post-ARDS morbidities.

Methods We analysed baseline variables and 6-month health status for participants in the ARDS Network LongTerm Outcomes Study. After division into derivation and validation datasets, we used weighted network analysis to identify subtypes from predictors and outcomes in the derivation dataset. We then used recursive partitioning to develop a subtype classification rule and assessed adequacy of the classification rule using a kappa statistic with the validation dataset.

Results Among 645 ARDS survivors, 430 were in the derivation and 215 in the validation datasets. Physical and mental health status, but not cognitive status, were closely associated. Four distinct subtypes were apparent (percentages in the derivation cohort): (1) mildly impaired physical and mental health ( $22 \%$ of patients), (2) moderately impaired physical and mental health (39\%), (3) severely impaired physical health with moderately impaired mental health (15\%) and (4) severely impaired physical and mental health (24\%). The classification rule had high agreement (kappa $=0.89$ in validation dataset). Female Latino smokers had the poorest status, while male, non-Latino non-smokers had the best status. Conclusions We identified four post-ARDS outcome subtypes that were predicted by sex, ethnicity, preARDS smoking status and other baseline factors. These subtypes may help develop tailored rehabilitation strategies, including investigation of combined physical and mental health interventions, and distinct interventions to improve cognitive outcomes.

\section{INTRODUCTION}

Recent advances have reduced short-term mortality for patients with acute respiratory distress syndrome (ARDS),${ }^{12}$ with an increasing focus on the postdischarge morbidities commonly experienced by ARDS survivors. ${ }^{3}{ }^{4}$ ARDS survivors frequently experience persistent impairments in physical (eg, muscle weakness), cognitive (eg, impaired memory) and mental health (eg, anxiety) status, coupled with reduced quality of life (QOL), ${ }^{5-7}$ known as the

\section{Key messages}

What is the key question?

- Are there subtypes of physical, cognitive and mental health outcomes among acute respiratory distress syndrome (ARDS) survivors?

What is the bottom line?

- Four distinct subtypes were apparent, generally based on similar severity levels for both physical and mental health impairments in ARDS survivors.

Why read on?

- To discover the patterns of subtypes, predictors of subtype membership and relationships among physical, cognitive and mental health outcomes among ARDS survivors.

postintensive care syndrome. ${ }^{89}$ Despite substantial epidemiological research describing impairments in these outcome domains, the patterns and co-occurrence across domains of impairment are poorly understood.

Some researchers suggest that survivors' post-ICU health status impairments represent not one syndrome but many. ${ }^{10}$ Distinct syndromes within larger clinical phenomena-when they include biological mechanisms paired with specific phenotypes-are commonly termed endotypes. ${ }^{11}$ The discovery of endotypes is a multistep process that begins with the identification of apparent subtypes (sometimes called subgroups or phenotypes). Those subtypes that ultimately prove to be associated with a biological mechanism are then considered endotypes. ${ }^{12}$ If multiple post-ARDS outcome endotypes exist, identifying such endotypes could facilitate research directed at understanding the pathophysiology, natural history and response to therapy within the larger pool of ARDS survivors. ${ }^{13}$ Identifying endotypes also may allow targeted studies of causal mechanisms and therapeutic interventions in a way that is not currently possible when evaluating more heterogeneous patient groups. ${ }^{14} 15$

Hence, to advance the understanding of postARDS patient outcomes, especially the associations among physical, cognitive and mental health status, we performed a cluster analysis of ARDS survivors 
Table 1 Health status outcome instruments employed in this study

\begin{tabular}{lll}
\hline Instrument & Domain measured & Items (N) \\
\hline EuroQOL (EQ-5D-3L) & Quality of life & 6 \\
$\begin{array}{l}\text { SF-36v2 Health Survey (includes a Mental } \\
\text { Component Summary and Physical Component } \\
\text { Summary) }\end{array}$ & $\begin{array}{l}\text { Physical and mental } \\
\text { health }\end{array}$ & 36 \\
$\begin{array}{l}\text { Functional Assessment of Chronic Illness } \\
\text { Therapy }\end{array}$ & Fatigue & 13 \\
$\begin{array}{l}\text { Telephone Mini Mental State Exam } \\
\text { Hospital Anxiety and Depression Scale }\end{array}$ & Cognitive status & 11 \\
$\begin{array}{l}\text { Impact of Event Scale-Revised (measure of } \\
\text { post-traumatic stress disorder symptoms) }\end{array}$ & Mental health & 22 \\
\begin{tabular}{l} 
Functional Performance Inventory - Short Form \\
\hline
\end{tabular} & Physical function & 32 \\
\hline
\end{tabular}

enrolled in a national, multicentre study to identify outcome subtypes. This work represents the first essential step in endotyping: the identification and definition of apparent subtypes among ARDS survivors.

\section{METHODS}

\section{Study design}

We conducted a separately funded and designed, data science analysis of patients in the ARDS Network Long-Term Outcomes Study (ALTOS), which prospectively collected posthospitalisation outcomes for ARDS survivors from three ARDS Network multicentre randomised trials, as described elsewhere. ${ }^{16-18}$ ALTOS enrolled participants from 41 hospitals throughout the USA. ${ }^{19} 20$ The parent trials, ALTOS, and the present study were approved by the relevant Institutional Review Boards, with appropriate informed consent from participants.

\section{Primary analysis}

For this study, the primary analysis was the identification and validation of outcome subtypes among ARDS survivors, using weighted network analysis (WNA), which incorporates information about baseline predictors (at the time of hospital admission) and outcome variables (measured at 6 months). We chose WNA over, for example, latent class analysis, specifically because WNA is well suited to bring together predictors and outcomes in a way that facilitates the identification of meaningful subtypes.

\section{Constituent patient outcomes}

As described previously, ${ }^{19} 21$ at 6 months, a comprehensive battery of validated outcome instruments was administered to study participants (table 1), which included measures of physical, cognitive, mental health and quality of life status.

\section{Statistical methods}

Subtyping through WNA in the derivation dataset

We employed WNA, a clustering technique originally developed for genetic analysis, to identify subtypes. WNA distinctively incorporates both baseline predictor and 6-month outcome variables in the clustering process. ${ }^{22}$ The process (detailed in online supplementary appendix 1) proceeds as follows: (1) identification of the most relevant predictors, (2) hierarchical clustering of those predictors, (3) adjustment of cluster definitions to match patterns in outcome data, (4) development of prediction rules for subtype membership and (5) validation of clustering and prediction.
We began this work with a penalised regression model of 6-month health utility scores obtained from the EQ-5D-3L QOL instrument, ${ }^{23-25}$ the results of which have been published. ${ }^{26}$ This first step allowed us to reduce the number of candidate predictors to facilitate subsequent clustering. That initial work identified nine predictors associated with 6-month health utility: age, sex, Latino ethnicity, current smoking at the time of hospital admission, body mass index, pulmonary comorbidity, AIDS comorbidity, nadir respiratory rate on the day of study enrolment and residential independence at time of hospital admission (ie, whether the patient resided at home with no help, at home with informal help or required either professional help at home or resided in a healthcare facility). ${ }^{22}$ The present work builds on, and differs from, that preliminary work by performing a cluster analysis of the limited predictor set and merging those clusters with the outcome data. The present work thus focuses on identifying subtypes among ARDS outcomes, characterising the subtypes and building prediction models for subtype membership. We dealt with the rare missing data in the trial dataset predictors, as outlined in our initial work, with multivariate single imputation before initiation of analysis; we did not impute any outcome data. ${ }^{27}$

In order to allow validation, we applied WNA to a derivation dataset consisting of a random selection of two-thirds $(n=430)$ of ALTOS patients, with the remaining one-third $(n=215)$ reserved for validation.

\section{Evaluation of the adequacy of subtyping in the derivation dataset}

We evaluated the adequacy of the WNA-based subtyping by exploring standardised differences in constituent outcomes (and baseline predictor variables) by subtype and by calculating the network module metrics of Langfelder et al. ${ }^{28}$

\section{Subtype classification}

Since our purpose was to identify outcome subtypes that can be predicted at the time of therapeutic decisions (eg, shortly after ARDS onset), we used recursive partitioning (RPART), ${ }^{29}$ a statistical learning technique, to define a classification rule for our subtypes. RPART generated a final set of predictor variables and cut-off values within the predictors to define our WNA-based subtypes. The WNA and RPART classification rule was developed in the derivation cohort, with the cluster structure and classification rule applied to the validation cohort (online supplementary appendix 1).

\section{Subtype validation}

Subtype membership can be determined in a validation dataset in two ways: (1) using correlation with the subtype pattern summaries from the derivation dataset, and (2) using the classification rule. Agreement between these approaches suggests that the subtypes are valid. We assigned cluster membership in the validation dataset by correlating each patient's profile across the predictor variable set with the first principal component of each WNA cluster in the derivation set, a WNA concept called 'module membership'. ${ }^{28} 30$ For each patient in the derivation dataset, we assigned subtype membership based on the cluster that it achieved maximal Pearson correlation with via the cluster's first principal component. Additionally, we applied the RPART classification rule that was created in the derivation cohort. We then compared the agreement of the WNA subtype membership with the RPART subtype membership in the validation dataset using a kappa score (online supplementary appendix 1). 


\section{Subtype description}

We descriptively compared outcomes and demographics across subtypes in the derivation and validation datasets (considered separately) using analysis of variance for comparing subtypes with continuous variables and $\chi^{2}$ or Fisher's exact tests for categorical variables.

To address the risk of type 1 error arising from multiple comparisons, we limited the false discovery rate to $<5 \%$ using the technique of Benjamini. ${ }^{31}$

\section{Secondary analyses}

Five secondary analyses were performed. First, as part of a National Heart Lung and Blood Institute (NHLBI)-funded prospective substudy within ALTOS, participants recruited from 5 of the 12 ALTOS study centres completed additional outcome evaluations, including a detailed battery of cognitive tests at 6-month follow-up and evaluation of pre-ARDS health status (retrospectively reported by patients using EQ-5D and SF-36). ${ }^{20} 32$ In this analysis, we combined patients from the derivation and validation datasets using RPART to assign subtype in both datasets and evaluated whether cognitive test scores differed by subtype and whether the cognitive test scores correlated with other outcomes (eg, mental and physical health) using Pearson correlations and inspection of the biplot from a principal components analysis of the cognitive outcome scores (online supplementary appendix 1).

Second, in the same subgroup of patients with additional detailed outcome assessment, we evaluated whether baseline EQ-5D and SF-36 scores, and change in scores from baseline to 6 months, differed by subtype. We also measured the proportion of survivors whose score decreased, from baseline, by more than the minimally important difference for EQ-5D (0.07 points $)^{33}$ and SF-36 (4 points) $)^{34} 35$ and to capture more extreme decrements in health status, those whose scores decreased by more than 1 SD on either instrument.

Third, we evaluated whether survivors' 6-month residential independence and their change in residential independence from baseline to 6-month follow-up differed by subtype in the derivation and validation datasets.

Fourth, we explored ethnicity-related and subtype-related differences in household income by residential zip code, educational attainment and baseline employment status (online supplementary appendix 1 ).

Finally, because Euclidean distance may not be optimal for multiple data types, we performed a sensitivity analysis using Gower's distance, ${ }^{36}$ which was developed to measure distance among multiple data types. We then compared the WNA clustering results based on Gower's distance to the Euclidean distance-derived clusters.

\section{Sample size}

WNA can be performed with sample sizes as small as $20-100 .^{2237-39}$ Given a sample size of 215 patients in the validation dataset and kappa estimates of 0.7 and 0.8 for agreement of the classification rule with the WNA results, the proposed analysis would have $95 \%$ CI widths of 0.132 and 0.094 , respectively, which was considered adequately precise ${ }^{40}$ (see online supplementary appendix 1).

We performed all analyses in R 3.2.3 (www.r-project.org). ${ }^{41}$ Statistical significance was assessed at the 0.05 level.

\section{RESULTS}

A total of 1176 eligible patients were enrolled in the ARDS Network clinical trials, with 698 survivors being eligible and consenting for ALTOS, of whom 42 (6\%) died prior to 6-month follow-up. A total of 645 (98\%) of the 656 eligible, consenting survivors completed 6-month follow-up (figure 1).

Results of WNA in the derivation cohort are depicted in online supplementary figure 1. WNA identified four subtypes, with the identified clusters meeting Langfelder et al's criteria for adequacy (online supplementary table 1). The four-cluster solution was more consistent with the patterns in WNA than possible three-cluster or five-cluster solutions. The standardised outcomes demonstrated good separation by subtype (figure 2), with statistically significant differences among the large majority of outcomes. Only cognitive function, as measured by MiniMental State Exam (MMSE), did not differ significantly across subtypes.

Physical and mental health status were closely associated, while cognitive outcomes were not (online supplementary tables 5 and 6). In three of four subtypes, we observed parity in the severity of physical and mental health status impairments, whether mild (subtype 1), moderate (subtype 2) or severe (subtype 4). We also identified a subtype (subtype 3 ) in which severe physical health status impairment was coupled with only moderate mental health status impairment.

Table 2 depicts the mean scores for each instrument subscale and total score, by subtype, in the derivation dataset. Among the baseline predictors used in the analysis (table 3), the subtypes differed significantly by age, sex, Latino ethnicity, pulmonary comorbidity, residential independence (as previously defined) and current tobacco smoking prior to hospital admission. For purposes of illustration, we also compared potentially relevant severity of illness variables across subtypes. The subtypes did not differ by sepsis as primary cause of ARDS, intensive care unit length of stay (ICU LOS), Acute Physiology and Chronic Health Evaluation score, arterial oxygen tension (or pressure) $\left(\mathrm{PaO}_{2}\right) /$ fractional inspired oxygen $\left(\mathrm{FiO}_{2}\right)$ ratio, positive end expiratory pressure, Glasgow Coma Scale score or presence of shock at enrolment (all $\mathrm{p}>0.1$ ).

The RPART classification rule based on the derivation dataset $(n=430)$ is depicted in online supplementary figure 2. The agreement for the RPART classification rule with the WNA subtypes was excellent (kappa 0.92, 95\% CI 0.89 to 0.95 ; online supplementary table 2). Baseline pulmonary comorbidity, sex, Latino ethnicity, residential status and current smoking status were the variables that drove classification into the four subtypes.

In the validation cohort, agreement for the RPART versus the WNA subtype assignment remained excellent (kappa $0.89,95 \%$ CI 0.83 to 0.94 ; online supplementary table 3 ). Prediction from RPART versus WNA matched in $92 \%$ of the 205 cases where WNA subtype assignment was possible (online supplementary table 3). The separation of outcome variables by subtype appeared similar between the derivation and validation datasets (online supplementary figure 3).

The secondary analyses demonstrated that outcomes from the battery of detailed cognitive tests did not differ by subtype (online supplementary table 4), as observed using the MMSE cognitive screening test in the full dataset. Although physical and mental health outcomes correlated with each other (online supplementary table 5), there was a low correlation between cognitive and other outcomes (mean correlation 0.18 ; online supplementary table 6 ), with our exploratory principal components analysis (online supplementary figure 4) demonstrating that cognitive outcomes were generally unrelated to the physical and mental health outcomes. This was true in the entire cohort, where cognitive function was 


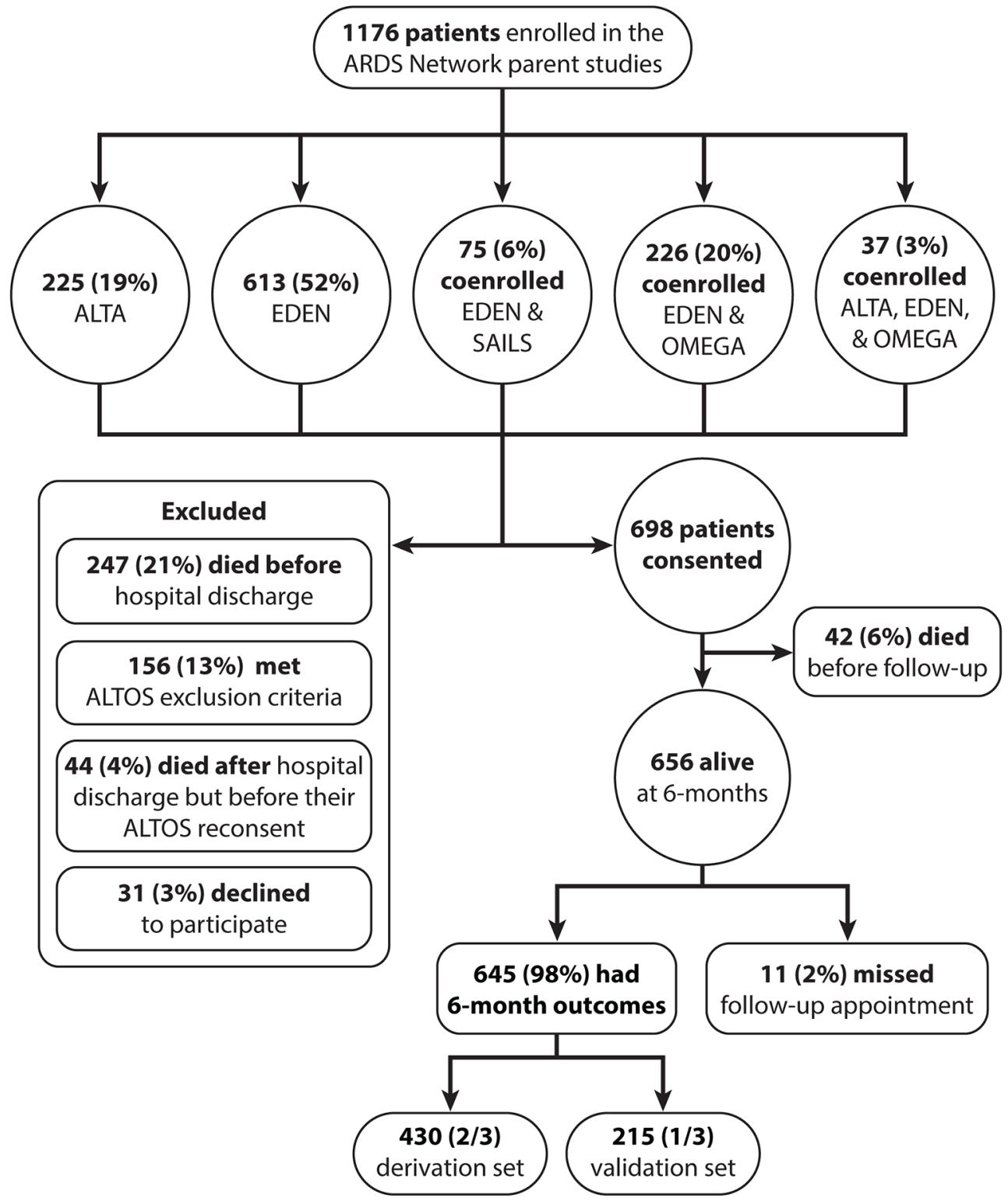

Figure 1 Patient flow diagram depicting the identification of patients for this study. ALTOS, ARDS Network Long-Term Outcomes Study; ARDS, acute respiratory distress syndrome.

measured by MMSE, and in the subgroup with additional detailed cognitive assessments.

Baseline health status differed significantly among subtypes (online supplementary table 7), with worse baseline health status associated with worse post-ARDS outcomes, as expected. However, patients in every subtype experienced a decrement from baseline status. Just under half of patients experienced a decrement that was larger than the minimum important difference in the EQ-5D health utility; a similar proportion of patients experienced a similar decrement in SF-36 physical component score. Approximately one-third of patients experienced a decrease in health status of at least 1 SD for the EQ-5D; one-third experienced a similar decrease in SF-36 (online supplementary table 7). The proportion of patients declining by more than the minimum important difference or by more than 1 SD for either outcome measure was similar across each of the four subtypes. This finding of decreased health status from baseline was reinforced by findings regarding residential independence. There was a marked decline in patients living at home without help from $91 \%$ at baseline to $45 \%$ at 6 -month follow-up, without significant differences in 6-month residential status across the four subtypes ( $p>0.1$; online supplementary table 8 ). The change in status was, however, significant.

In exploring socioeconomic status by ethnicity (ie, Latino vs non-Latino), we observed no significant difference in median income for home zip code (online supplementary table 9). Employment status did not differ by ethnicity (online supplementary table 10), although Latino patients did, on average, have more physically demanding occupations $(55 \%$ vs $33 \%, p=0.02)$. In the patient subgroup with additional detailed outcome assessments $(n=181)$, Latino patients had on average 4 years less educational attainment $(\mathrm{p}=0.01$; online supplementary table 11$)$.

In our sensitivity analysis using Gower's distance, the WNA clusters appeared similar to the WNA clusters identified by Euclidean distance. 


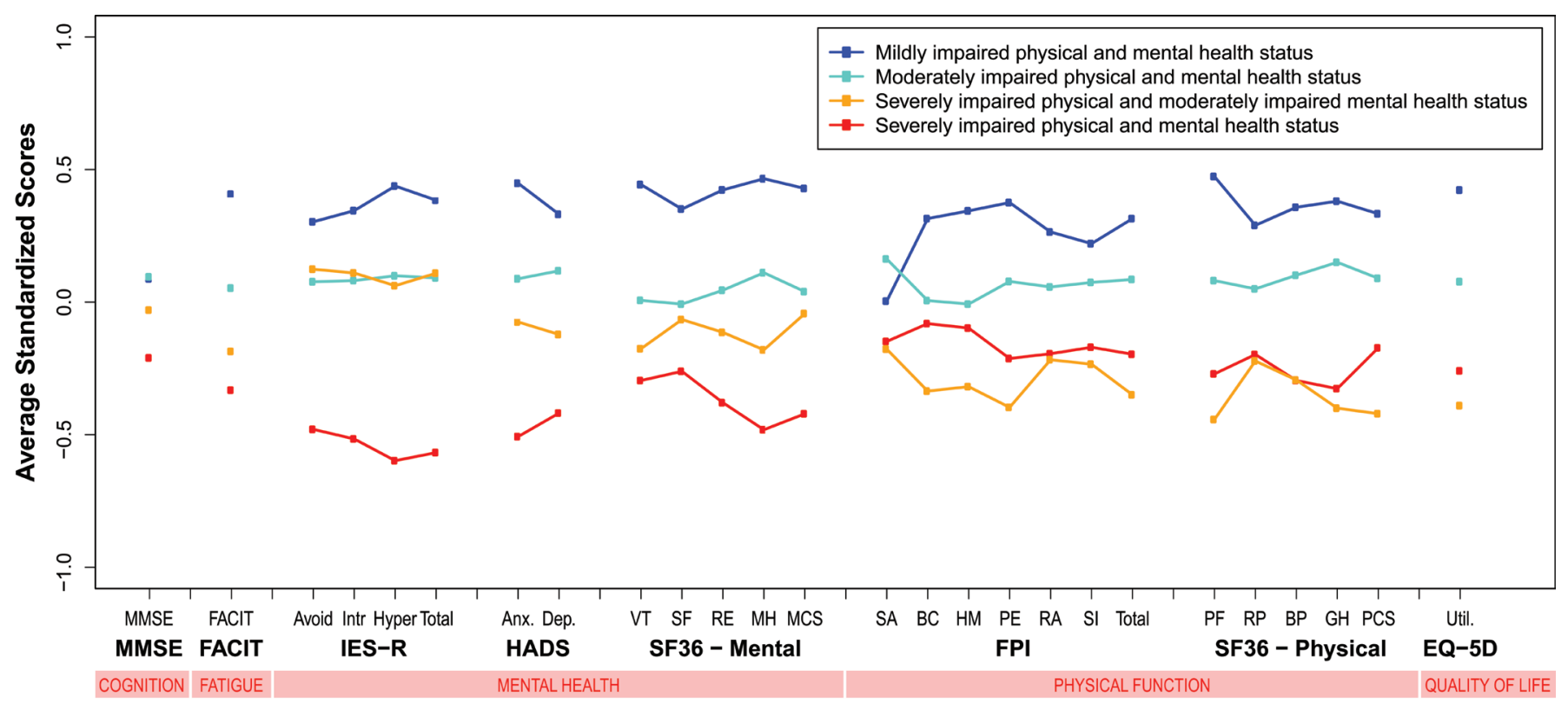

Figure 2 Average standardised outcomes by subtype, derivation dataset. The scores are normalised and standardised. Each subtype is represented by its own line, and the figure depicts the differences among the subtypes. EQ-5D: EuroQol; FACIT, Functional Assessment of Chronic Illness TherapyFatigue; FPI: Functional Performance Inventory; HADS, Hospital Anxiety and Depression Scale; IES-R; Impact of Event Scale, Revised; MMSE, Mini Mental State Examination; SF-36: Optum SF-36v2 Health Survey.

\section{DISCUSSION}

In this national, multicentre, prospective study of ARDS survivors, we identified four subtypes based on baseline predictors and health status at 6 months. Physical and mental health status were closely associated with each other, but cognitive status was not associated with these other outcomes. The most common subtype (39\% of patients) was moderately impaired physical and mental health status, with severe and mild impairments in both physical and mental health status representing 22\% and 23\% of patients, respectively. A third of patients suffered a decrease from baseline in their EQ-5D or SF-36 scores that was larger than $1 \mathrm{SD}$, a decrease that was similar across all four subtypes. These four post-ARDS outcome subtypes were predicted by sex, ethnicity and pre-ARDS smoking status and other baseline factors but were not predicted by acute ARDS severity or ICU treatment-related variables. Female Latino smokers had the most severe impairments, while male non-Latino non-smokers had the least severe impairments. These identified subtypes, as a foundation for identifying biologically based ARDS endotypes, may help inform the design of future interventions (acknowledging that the average effects in prior trials may have missed important effects in subgroups), including evaluating combined physical and mental health interventions. In addition, recognising the lack of correlation between cognitive and other outcomes, distinct interventions focused on improving cognitive impairment need to be evaluated. On the basis of current evidence, female smokers of Latina ethnicity are at especially high risk and merit further investigation of potential early interventions.

The lack of correlation between cognitive and non-cognitive (ie, physical and mental health) outcomes was consistent when both the MMSE screening instrument was used to evaluated cognitive outcomes in the entire population and when a detailed battery of cognitive tests was evaluated in an a priori subgroup of patients. Our findings extend recent findings from a national Canadian cohort of 463 patients who were mechanically ventilated for at least 1 week. This Canadian study observed that predictors of physical impairment were not predictive of cognitive impairment (both evaluated using the Functional Independence Measure). ${ }^{42}$ Our findings differ from a post hoc subgroup analysis of a prior study of 122 ARDS survivors that suggested (unadjusted $\mathrm{p}=0.04$ ) a possible association between anxiety and cognitive impairment. Our findings may suggest that the mechanisms of, or individual susceptibility for, cognitive impairment after ARDS are not related to those associated with physical and mental health impairments. Our data should not, however, be interpreted to suggest that nominally physical interventions, such as early mobilisation, will not improve cognitive outcomes, given the potential association between early mobilisation and delirium. Nor do our data suggest that some ARDS survivors require no preventive or rehabilitation interventions, only that tailoring such support may be important.

Patients in the subtype with severe physical and mental health impairments (subtype 4) were disproportionately female, current smokers at the time of hospital admission and Latino. This finding regarding Latino ethnicity may be at least in part a marker of baseline socioeconomic differences, as our exploratory analyses suggested that Latino versus non-Latino patients had lower educational attainment and a greater proportion had physically demanding employment.

The present work builds on our prior preliminary work ${ }^{26}$ in several ways. While our prior work ${ }^{26}$ identified risk factors for poor health-related QOL, the present work identifies subtypes of outcomes and builds a prediction rule to assign patients to a given outcome subtype. The present work also investigates the apparent distinction of cognitive outcomes from non-cognitive outcomes and further explores the relevance of ethnicity to postARDS outcome subtypes.

The predictors we identified as relevant differed from the previously mentioned Canadian study, which found that older age and longer ICU LOS were important predictors of worse physical outcomes among survivors. ${ }^{42}$ Differences in patient populations may explain, at least partly, the differences in findings, including our specific focus on ARDS patients with higher severity of illness at the time of ICU admission, as well as other 
Table 2 Distribution of outcomes by subtype in the derivation dataset (mean (SD) or N (\%))

\begin{tabular}{|c|c|c|c|c|c|c|}
\hline Instrument* & $\begin{array}{l}\text { Overall } \\
(n=430) \\
\text { Mean (SD) or N (\%) }\end{array}$ & $\begin{array}{l}\text { Mildly impaired } \\
\text { physical and mental } \\
\text { health status ( } n=94 \\
(22 \%)) \\
\text { Mean (SD) or } N(\%)\end{array}$ & $\begin{array}{l}\text { Moderately impaired } \\
\text { physical and mental } \\
\text { health status }(n=166 \\
(39 \%)) \text { Mean (SD) or } \\
N(\%)\end{array}$ & $\begin{array}{l}\text { Severely impaired } \\
\text { physical and } \\
\text { moderately impaired } \\
\text { mental health status } \\
(\mathrm{n}=66(15 \%)) \text { Mean } \\
(\mathrm{SD}) \text { or N }(\%)\end{array}$ & $\begin{array}{l}\text { Severely impaired } \\
\text { physical and mental } \\
\text { health status }(n=104 \\
(24 \%)) \text { Mean (SD) or } \\
N(\%)\end{array}$ & $\begin{array}{l}p \text { value (adjusted } \\
\text { for multiple } \\
\text { comparisons) }\end{array}$ \\
\hline \multicolumn{7}{|l|}{ MMSE (cognition) } \\
\hline Score (points) & $25(2)$ & $26(2)$ & $26(2)$ & $25(1)$ & $25(2)$ & 0.09 \\
\hline \multicolumn{7}{|l|}{ FACIT (fatigue) } \\
\hline Score (points) & $60(17)$ & $67(16)$ & $61(18)$ & $57(12)$ & $54(17)$ & $<0.001$ \\
\hline $\begin{array}{l}\text { Abnormal FACIT } \\
(\leq 68)\end{array}$ & $293(72)$ & $47(53)$ & $114(72)$ & $51(81)$ & $81(83)$ & $<0.001$ \\
\hline $\begin{array}{l}\text { Hyperarousal } \\
\text { subscale score }\end{array}$ & $0.9(1.0)$ & $0.5(0.6)$ & $0.8(0.9)$ & $0.9(0.8)$ & $1.5(1.1)$ & $<0.001$ \\
\hline Total score & $1.0(0.9)$ & $0.7(0.7)$ & $0.9(0.8)$ & $0.9(0.8)$ & $1.5(1.0)$ & $<0.001$ \\
\hline $\begin{array}{l}\text { Abnormal IES-R } \\
(\geq 1.6)\end{array}$ & $97(24)$ & $9(10)$ & $30(19)$ & $14(22)$ & $44(45)$ & $<0.001$ \\
\hline \multicolumn{7}{|c|}{ HADS (anxiety and depression symptoms) } \\
\hline $\begin{array}{l}\text { Anxiety subscale } \\
\text { score }\end{array}$ & $7(5)$ & $5(4)$ & $7(4)$ & $7(5)$ & $10(5)$ & $<0.001$ \\
\hline $\begin{array}{l}\text { Abnormal HADS-A } \\
(\geq 8)\end{array}$ & $168(41)$ & $20(22)$ & $61(38)$ & $28(44)$ & $59(61)$ & $<0.001$ \\
\hline \multicolumn{7}{|c|}{ SF-36 v2 - Mental Health (normalised) } \\
\hline Social role scale & $41(14)$ & $46(13)$ & $41(14)$ & $40(14)$ & $37(15)$ & 0.001 \\
\hline Emotional role scale & $41(15)$ & $48(13)$ & $42(15)$ & $39(15)$ & $35(15)$ & $<0.001$ \\
\hline Mental health scale & $44(14)$ & $51(12)$ & $46(13)$ & $42(13)$ & $37(15)$ & $<0.001$ \\
\hline $\begin{array}{l}\text { Mental component } \\
\text { Summary (MCS) } \\
\text { Score }\end{array}$ & $45(15)$ & $51(13)$ & $46(13)$ & $45(14)$ & $39(16)$ & $<0.001$ \\
\hline Abnormal MCS $(<35)$ & $105(25)$ & $13(15)$ & $37(23)$ & $17(27)$ & $38(38)$ & 0.002 \\
\hline \multicolumn{7}{|c|}{ FPI-Short Form (physical function) } \\
\hline $\begin{array}{l}\text { Spiritual activities } \\
\text { scale }\end{array}$ & $1.8(1.1)$ & $1.8(1.2)$ & $2.0(1.1)$ & $1.6(1.2)$ & $1.6(1.1)$ & 0.038 \\
\hline Body care scale & $2.5(0.7)$ & $2.7(0.5)$ & $2.5(0.7)$ & $2.3(0.8)$ & $2.5(0.7)$ & 0.001 \\
\hline $\begin{array}{l}\text { Household } \\
\text { maintenance scale }\end{array}$ & $1.8(0.9)$ & $2.1(0.9)$ & $1.8(0.9)$ & $1.5(0.8)$ & $1.7(0.8)$ & $<0.001$ \\
\hline $\begin{array}{l}\text { Physical exercise } \\
\text { scale }\end{array}$ & $1.5(0.9)$ & $1.9(0.8)$ & $1.6(0.9)$ & $1.2(0.8)$ & $1.4(0.8)$ & $<0.001$ \\
\hline Recreation scale & $2.0(0.9)$ & $2.2(0.9)$ & $2.0(0.8)$ & $1.8(0.8)$ & $1.8(0.8)$ & 0.003 \\
\hline Social activities scale & $1.8(1.0)$ & $2.0(0.9)$ & $1.9(1.0)$ & $1.6(1.0)$ & $1.6(0.9)$ & 0.008 \\
\hline Total score & $1.9(0.7)$ & $2.1(0.7)$ & $2.0(0.7)$ & $1.7(0.7)$ & $1.8(0.6)$ & $<0.001$ \\
\hline \multicolumn{7}{|c|}{ SF-36 v2 - physical health (normalised) } \\
\hline $\begin{array}{l}\text { Physical functioning } \\
\text { scale }\end{array}$ & $36(13)$ & $42(12)$ & $37(14)$ & $30(10)$ & $33(12)$ & $<0.001$ \\
\hline
\end{tabular}




\begin{tabular}{|c|c|c|c|c|c|c|}
\hline Instrument* & $\begin{array}{l}\text { Overall } \\
(n=430) \\
\text { Mean (SD) or N (\%) }\end{array}$ & $\begin{array}{l}\text { Mildly impaired } \\
\text { physical and mental } \\
\text { health status }(n=94 \\
(22 \%)) \\
\text { Mean (SD) or N }(\%)\end{array}$ & $\begin{array}{l}\text { Moderately impaired } \\
\text { physical and mental } \\
\text { health status }(n=166 \\
(39 \%)) \text { Mean (SD) or } \\
N(\%)\end{array}$ & $\begin{array}{l}\text { Severely impaired } \\
\text { physical and } \\
\text { moderately impaired } \\
\text { mental health status } \\
(n=66(15 \%)) \text { Mean } \\
(\text { SD) or N (\%) }\end{array}$ & $\begin{array}{l}\text { Severely impaired } \\
\text { physical and mental } \\
\text { health status }(n=104 \\
(24 \%)) \text { Mean (SD) or } \\
N(\%)\end{array}$ & $\begin{array}{l}p \text { value (adjusted } \\
\text { for multiple } \\
\text { comparisons) }\end{array}$ \\
\hline Physical role scale & $37(13)$ & $41(13)$ & $38(13)$ & $34(12)$ & $35(12)$ & 0.002 \\
\hline Bodily pain scale & $43(12)$ & $47(12)$ & $44(12)$ & $39(11)$ & $39(11)$ & $<0.001$ \\
\hline General health scale & $40(13)$ & $45(12)$ & $42(12)$ & $35(12)$ & $36(13)$ & $<0.001$ \\
\hline $\begin{array}{l}\text { Physical Component } \\
\text { Summary (PCS) score }\end{array}$ & $38(12)$ & $42(11)$ & $39(13)$ & $33(10)$ & $36(11)$ & $<0.001$ \\
\hline Abnormal PCS $(<35)$ & $191(46)$ & $25(28)$ & $74(46)$ & $39(61)$ & $53(54)$ & $<0.001$ \\
\hline \multicolumn{7}{|l|}{ EQ-5D (quality of life) } \\
\hline Utility & $0.69(0.24)$ & $0.79(0.19)$ & $0.71(0.23)$ & $0.60(0.24)$ & $0.63(0.24)$ & $<0.001$ \\
\hline \multicolumn{7}{|c|}{ 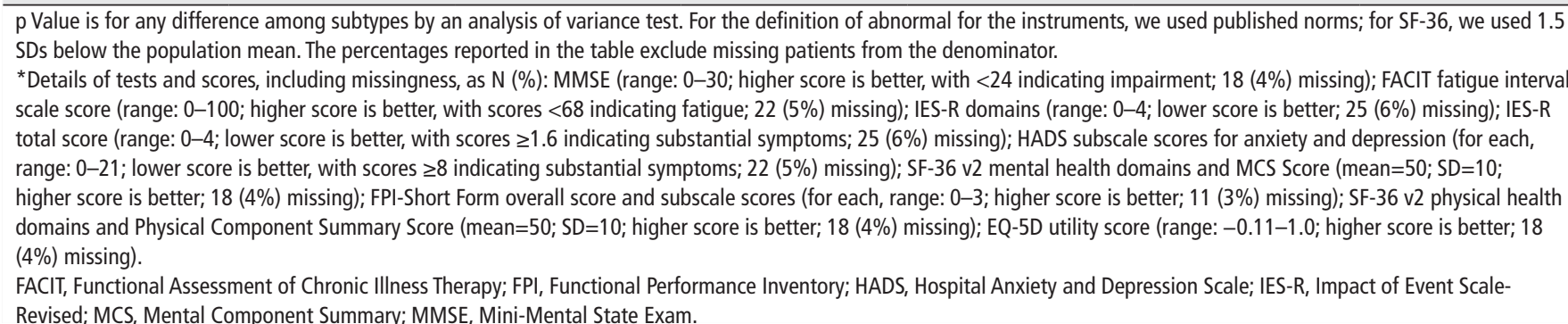 } \\
\hline
\end{tabular}

patient and health system differences reflected by a much shorter mean hospital LOS in our study (23 vs 49 days).

Among routinely available baseline data, demographic and lifestyle attributes present at hospital admission were useful in identifying four distinct subtypes at 6 months post-ARDS. A subgroup analysis also demonstrated that baseline health status was associated with subsequent 6-month status. However, this latter finding does not mean that postdischarge impairments simply reflect patients' pre-existing baseline status, since across all four subtypes, there was a substantial decrement in QOL, with one-third of patients having a decreased of $>1$ SD. As corroborative evidence, we also found that the percentage of patients living at home independently decreased from 91\% at baseline to $45 \%$ at 6 months, with similar resulting 6 -month residential status across all four subtypes. These findings are consistent with studies of sepsis survivors reporting decreased health status, ${ }^{43}$ or a change in health trajectories. ${ }^{44}$ Hence, while baseline status is an important predictor of post-ARDS outcomes, across all four subtypes, there was a large decline in health status and residential independence after ARDS experienced by a substantial proportion of patients.

Multiple factors likely explain the observation that markers of acute disease severity had little association with post-ARDS health status after hospital discharge. ${ }^{45-48}$ Acute severity of illness strongly predicts death: the patients who would have had the most severe impairments have no outcome assessment as a result of censoring due to death, given the $21 \%$ inpatient mortality rate. ${ }^{49}$ This inpatient mortality may also explain the lack of association between age and outcome. Moreover, among survivors, ARDS may be such a severe physiological insult that gradations of severity within ARDS do not result in differences in health status afterwards. We acknowledge that we were unable to measure incident delirium, which could be associated with long-term outcomes, especially cognitive impairment. We also acknowledge that we had detailed cognitive data only on a subset of our patient population; a link between acute severity of illness and cognition, perhaps mediated by delirium, ${ }^{50}$ cannot be excluded.

Among the four subtypes, there was only one group, representing $15 \%$ of all patients, in which the severity of physical and mental impairment was not very closely aligned, with patients having severe physical impairment and only moderate mental health impairment. It is possible that these individuals were more resilient, ${ }^{51}$ or that they had pre-existing physical illness that affected their adaptation to post-ARDS physical outcomes. The latter explanation may be supported by these patients being older and less likely to reside independently at home than patients in the subtype with severe physical and severe mental impairment. However, there were no differences in baseline EQ-5D or SF-36 between these subtypes, so further prospective evaluation is needed.

We acknowledge that we have identified subtypes in this work, but they may not ultimately resolve to endotypes based on biological mechanisms. While the outcome measures are widely validated in multiple disease states, the relevant physiological parameters may not be adequately represented by our outcome measures. Nevertheless, our findings may have relevance in advancing the goals of personalised medicine for patients with ARDS. ${ }^{52} 53$ Several randomised controlled trials of ICU and post-ICU rehabilitation interventions have demonstrated improvements in some outcomes, but not in others, ${ }^{5455}$ suggesting a need to identify and target patient subgroups that may be maximally responsive to a given intervention $^{56}$ and to consider multimodal interventions.

This analysis has important strengths, including its large sample size; national, multicentre design; and very high rates of follow-up to minimise selection bias. However, there are also potential limitations. First, baseline QOL and functional status (including mental health and cognitive status) cannot be obtained prospectively in patients with ARDS, leading to potential recall bias affecting our subgroup analyses. However, these subgroup results were similar when analysing changes in baseline residential independence, where residential independence may be a measure of baseline health status less subject to recall 
Table 3 Distribution of predictor variables by outcome subtype in the derivation dataset

\begin{tabular}{|c|c|c|c|c|c|c|}
\hline $\begin{array}{l}\text { Predictor variable } \\
\left(\text { Mean }(\text { SD) or N }(\%))^{*}\right.\end{array}$ & $\begin{array}{l}\text { Overall } \\
(\mathrm{n}=430)\end{array}$ & $\begin{array}{l}\text { Mildly impaired } \\
\text { physical and mental } \\
\text { health status }(n=94)\end{array}$ & $\begin{array}{l}\text { Moderately impaired } \\
\text { physical and mental } \\
\text { health status ( } n=166 \text { ) }\end{array}$ & $\begin{array}{l}\text { Severely impaired } \\
\text { physical and } \\
\text { moderately impaired } \\
\text { mental health status } \\
(n=66)\end{array}$ & $\begin{array}{l}\text { Severely } \\
\text { impaired } \\
\text { physical } \\
\text { and mental } \\
\text { health } \\
\text { status } \\
(n=104) \\
\end{array}$ & $\begin{array}{l}\mathbf{p} \\
\text { value (adjusted } \\
\text { for multiple } \\
\text { comparisons) }\end{array}$ \\
\hline Age & 49 (14) & $48(17)$ & 49 (15) & 54 (13) & 47 (11) & 0.01 \\
\hline Nadir respiratory rate & $18(6)$ & $19(6)$ & $18(6)$ & $17(6)$ & $19(7)$ & 0.09 \\
\hline Body mass index & $30(8)$ & $30(7)$ & $30(7)$ & $31(8)$ & $31(9)$ & 0.60 \\
\hline AIDS comorbidity & $6(1)$ & $3(3)$ & $2(1)$ & $0(0)$ & $1(1)$ & 0.51 \\
\hline Pulmonary comorbidity & $47(11)$ & $0(0)$ & $1(1)$ & $42(64)$ & $4(4)$ & $<0.001$ \\
\hline $\begin{array}{l}\text { Requires professional help at home } O R \text { residence } \\
\text { in healthcare facility }\end{array}$ & $14(3)$ & $0(0)$ & $14(8)$ & $0(0)$ & $0(0)$ & \\
\hline \multicolumn{7}{|l|}{$\begin{array}{l}\text { Acute illness factors, depicted for purposes of } \\
\text { illustration }\end{array}$} \\
\hline Sepsis as cause of ARDS $\ddagger$ & $334(78)$ & $66(70)$ & $129(78)$ & $52(79)$ & $87(84)$ & 0.16 \\
\hline ICU LOS & $14(11)$ & $14(9)$ & $15(12)$ & $14(11)$ & $15(11)$ & 0.75 \\
\hline APACHE III & $85(25)$ & $86(25)$ & $84(24)$ & $90(25)$ & $84(27)$ & 0.41 \\
\hline PEEP & $10(4)$ & $10(4)$ & $9(4)$ & $9(3)$ & $10(4)$ & 0.16 \\
\hline Minimum PF & $116(54)$ & $122(59)$ & $115(55)$ & $118(50)$ & $110(50)$ & 0.41 \\
\hline GCS & $8(3)$ & $8(4)$ & $9(3)$ & $9(3)$ & $8(4)$ & 0.84 \\
\hline
\end{tabular}

*Percentages reported are on the basis of non-missing values.

tAt time of hospital admission for ARDS.

¥Either sepsis or pneumonia as primary cause of ARDS.

APACHE III, Acute Physiology and Chronic Health Evaluation score; ARDS, acute respiratory distress syndrome; GCS, Glasgow Coma Scale score; ICU LOS: intensive care unit length of stay; PEEP, positive End expiratory pressure; $\mathrm{PF}$, arterial oxygen tension (or pressure): ifractional inspired oxygen ratio

bias. Second, only survivors can be included in analyses of health status outcomes. However, the low mortality rate after discharge may limit such bias arising from censorship due to death, at least among hospital survivors. Third, our study population was ARDS survivors from large-scale clinical trials with strict inclusion and exclusion criteria, which may limit generalisability to general ICU populations or even patients with ARDS outside research centres. Fourth, while we used separate derivation and validation datasets, we did not employ a wholly external dataset for validation; future research should include external validation. Fifth, while the instruments are well validated in large cohorts, it is possible that some of the association with Latino ethnicity could be mediated by ethnic differences in responses to instruments. We think this is unlikely given extensive prior validation of these instruments but cannot exclude it entirely.

\section{CONCLUSION}

In this national, prospective study of ARDS survivors, we identified four post-ARDS outcome subtypes based on baseline predictors and health status outcomes at 6 months. Physical health and mental health impairments were closely associated with each other, while cognitive impairment was not associated with either. Forty-one per cent of patients were in the moderate physical and mental health impairment subtype. Patients in every subtype suffered decreases from baseline health status. Outcome subtype membership was predicted by sex, ethnicity and pre-ARDS smoking status, with female Latino smokers having the poorest status, and male non-Latino non-smokers having the best status. This information can help inform the design of future interventions to improve post-ARDS patient outcomes, including evaluations of combined physical and mental health interventions as well as distinct interventions to improve cognitive outcomes.

\section{Author affiliations}

${ }^{1}$ Center for Humanizing Critical Care, Intermountain Healthcare, Murray, Utah, USA

${ }^{2}$ Department of Medicine, Pulmonary and Critical Care Division, Intermountain Medical Center, Murray, Utah, USA

${ }^{3}$ Pulmonary and Critical Care, University of Utah School of Medicine, Salt Lake City, Utah, USA

${ }^{4}$ Study Design and Biostatistics Center and Division of Epidemiology, University of Utah School of Medicine, Salt Lake City, Utah, USA

${ }^{5}$ Outcomes After Critical Illness and Surgery Group, Johns Hopkins University, Baltimore, Maryland, USA

${ }^{6}$ Division of Pulmonary and Critical Care Medicine, School of Medicine, Johns Hopkins University, Baltimore, Maryland, USA

${ }^{7}$ Department of Psychology and Neuroscience Center, Brigham Young University, Provo, Utah, USA

${ }^{8}$ Department of Physical Medicine and Rehabilitation, School of Medicine, Johns Hopkins University, Baltimore, Maryland, USA 
Twitter Follow Samuel M Brown @DrSamuelBrown

Contributors SMB, ROH, APP, TG and DMN designed the study. SMB, APP, TG, VDD, DMN and ELW analysed and interpreted the data. SMB, ROH and DMN drafted the report, and all other authors revised it. All authors gave final approval of the report to be published.

Funding National Heart Lung and Blood Institute (R21HL123433, N01HR56170, R01HL091760, R01HL091760:02S1, and contracts HHSN268200536165C to HHSN268200536175C and HHSN268200536179C).

Competing interests None declared.

Patient consent Obtained.

Ethics approval All procedures performed in studies involving human participants were in accordance with the ethical standards of the institutional and/or national research committee and with the 1964 Helsinki Declaration and its later amendments or comparable ethical standards.

Provenance and peer review Not commissioned; externally peer reviewed.

Data sharing statement In order to protect patient privacy and comply with relevant regulations, identified data are unavailable. Requests for deidentified data from qualified researchers with appropriate ethics board approvals and relevant data use agreements will be processed by the Intermountain Office of Research, officeofresearch@imail.org.

(c) Article author(s) (or their employer(s) unless otherwise stated in the text of the article) 2017. All rights reserved. No commercial use is permitted unless otherwise expressly granted.

\section{REFERENCES}

1 Ranieri VM, Rubenfeld GD, Thompson BT, et al. Acute respiratory distress syndrome: the Berlin Definition. JAMA 2012;307:2526-33.

2 Erickson SE, Martin GS, Davis JL, et al. Recent trends in acute lung injury mortality: 1996-2005. Crit Care Med 2009:37:1574-9.

3 Herridge MS, Tansey CM, Matté A, et al. Functional disability 5 years after acute respiratory distress syndrome. N Engl J Med 2011;364:1293-304.

4 Deutschman CS, Ahrens T, Cairns CB, et al. Multisociety Task Force for Critical Care Research: key issues and recommendations. Crit Care Med 2012;40:254-60.

5 Herridge MS, Cheung AM, Tansey CM, et al. One-year outcomes in survivors of the acute respiratory distress syndrome. N Engl J Med 2003;348:683-93.

6 Davydow DS, Desai SV, Needham DM, et al. Psychiatric morbidity in survivors of the acute respiratory distress syndrome: a systematic review. Psychosom Med 2008:70:512-9.

7 Herridge MS, Moss M, Hough CL, et al. Recovery and outcomes after the acute respiratory distress syndrome (ARDS) in patients and their family caregivers. Intensive Care Med 2016:42:725-38.

8 Elliott D, Davidson JE, Harvey MA, et al. Exploring the scope of post-intensive care syndrome therapy and care: engagement of non-critical care providers and survivors in a second stakeholders meeting. Crit Care Med 2014;42:2518-26.

9 Needham DM, Davidson J, Cohen $\mathrm{H}$, et al. Improving long-term outcomes after discharge from intensive care unit: report from a stakeholders' conference. Crit Care Med 2012:40:502-9.

10 Griffiths RD. Rehabilitating the critically ill: a cultural shift in intensive care unit care. Crit Care Med 2012:40:681-2.

11 Lötvall J, Akdis CA, Bacharier LB, et al. Asthma endotypes: a new approach to classification of disease entities within the asthma syndrome. J Allergy Clin Immunol 2011;127:355-60.

12 Russell CD, Baillie JK. Treatable traits and therapeutic targets: goals for systems biology in infectious disease. Curr Opin Syst Biol 2017;2:140-6.

13 Jameson JL, Longo DL. Precision medicine--personalized, problematic, and promising N Engl J Med 2015:372:2229-34.

14 Herridge M, Cameron J. Disability after critical illness. N Eng/ J Med 2013:369:1367-9.

15 Pearmain L, Herridge MS. Outcomes after ARDS: a distinct group in the spectrum of disability after complex and protracted critical illness. Minerva Anestesiol 2013;79:793-803.

16 Matthay MA, Brower RG, Carson S, et al. Randomized, placebo-controlled clinical trial of an aerosolized $\beta \square$-agonist for treatment of acute lung injury. Am J Respir Crit Care Med 2011;184:561-8.

17 Rice TW, Wheeler AP, Thompson BT, et al. Initial trophic vs full enteral feeding in patients with acute lung injury: the EDEN randomized trial. JAMA 2012:307:795-803

18 Rice TW, Wheeler AP, Thompson BT, et al. Enteral omega-3 fatty acid, gammalinolenic acid, and antioxidant supplementation in acute lung injury. JAMA 2011;306:1574-81.

19 Needham DM, Dinglas VD, Bienvenu OJ, et al. One year outcomes in patients with acute lung injury randomised to initial trophic or full enteral feeding: prospective follow-up of EDEN randomised trial. BMJ 2013;346:f1532.
20 Needham DM, Dinglas VD, Morris PE, et al. Physical and cognitive performance of patients with acute lung injury 1 year after initial trophic versus full enteral feeding. EDEN trial follow-up. Am J Respir Crit Care Med 2013;188:567-76.

21 Dinglas VD, Hopkins RO, Wozniak AW, et al. One-year outcomes of rosuvastatin versus placebo in sepsis-associated acute respiratory distress syndrome: prospective followup of SAILS randomised trial. Thorax 2016;71:401-10.

22 Horvath S. Weighted Network analysis: applications in genomics and Systems Biology: Springer, 2011

23 EuroQol Group. EuroQol--a new facility for the measurement of health-related quality of life. Health Policy 1990;16:199-208.

24 Turnbull AE, Rabiee A, Davis WE, et al. Outcome Measurement in ICU Survivorship Research from 1970 to 2013: a scoping review of 425 Publications. Crit Care Med 2016:44:1267-77.

25 Dowdy DW, Eid MP, Sedrakyan A, et al. Quality of life in adult survivors of critical illness: a systematic review of the literature. Intensive Care Med 2005;31:611-20.

26 Brown SM, Wilson E, Presson AP, et al. Predictors of 6-month health utility outcomes in survivors of acute respiratory distress syndrome. Thorax 2017:72.

27 Brown SM, Wilson E, Presson AP, et al. Predictors of 6-month health utility outcomes in survivors of acute respiratory distress syndrome. Thorax 2017;72:311-7.

28 Langfelder $\mathrm{P}$, Luo R, Oldham MC, et al. Is my network module preserved and reproducible? PLoS Comput Biol 2011;7:e1001057

29 Breiman L, Friedman J, Olshen R, et al. Classification and regression trees. Belmont, CA: Wadsworth, 1984.

30 Langfelder $P$, Horvath S. WGCNA: an R package for weighted correlation network analysis. BMC Bioinformatics 2008;9:559.

31 Benjamini Y, Hochberg Y. Controlling the False Discovery Rate: A Practical and Powerful Approach to Multiple Testing. J R Stat Soc Series B 1995;57:289-300.

32 Needham DM, Colantuoni E, Dinglas VD, et al. Rosuvastatin versus placebo for delirium in intensive care and subsequent cognitive impairment in patients with sepsis-associated acute respiratory distress syndrome: an ancillary study to a randomised controlled trial. Lancet Respir Med 2016;4:203-12.

33 Pickard AS, Neary MP, Cella D. Estimation of minimally important differences in EQ-5D utility and VAS scores in Cancer. Health Qual Life Outcomes 2007;5:70.

34 Samsa G, Edelman D, Rothman ML, et al. Determining clinically important differences in health status measures: a general approach with illustration to the Health Utilities Index Mark II. Pharmacoeconomics 1999;15:141-55.

35 Hays RD, Morales LS. The RAND-36 measure of health-related quality of life. Ann Med 2001;33:350-7.

36 Gower JC. A General Coefficient of Similarity and some of its Properties. Biometrics 1971;27:857-71.

37 Presson AP, Sobel EM, Papp JC, et al. Integrated weighted gene co-expression network analysis with an application to chronic fatigue syndrome. BMC Syst Biol 2008;2:95

38 Presson AP, Yoon NK, Bagryanova L, et al. Protein expression based multimarker analysis of breast Cancer samples. BMC Cancer 2011;11:230.

39 Levine AJ, Miller JA, Shapshak P, et al. Systems analysis of human brain gene expression: mechanisms for HIV-associated neurocognitive impairment and common pathways with Alzheimer's disease. BMC Med Genomics 2013:6:4

40 Walter SD, Eliasziw M, Donner A. Sample size and optimal designs for reliability studies. Stat Med 1998:17:101-10.

41 R Foundation for Statistical Computing. R: a language and environment for Statistical Computing. Vienna, Austria: R Foundation for Statistical Computing, 2009.

42 Herridge MS, Chu LM, Matte A, et al. The RECOVER program: disability risk groups and 1-Year outcome after 7 or more days of mechanical ventilation. Am J Respir Crit Care Med 2016;194:831-44.

43 Barnato AE, Albert SM, Angus DC, et al. Disability among elderly survivors of mechanical ventilation. Am J Respir Crit Care Med 2011;183:1037-42.

44 Iwashyna TJ, Netzer G, Langa KM, et al. Spurious inferences about long-term outcomes: the case of severe Sepsis and geriatric conditions. Am J Respir Crit Care Med 2012;185:835-41

45 Oeyen SG, Benoit DD, Annemans L, et al. Long-term outcomes and quality of life in critically ill patients with hematological or solid malignancies: a single center study. Intensive Care Med 2013:39:889-98.

46 Nisula S, Vaara ST, Kaukonen KM, et al. Six-month survival and quality of life of intensive care patients with acute kidney injury. Crit Care 2013;17:R250.

47 Hofhuis JG, Spronk PE, van Stel HF, et al. Quality of life before intensive care unit admission is a predictor of survival. Crit Care 2007;11:R78.

48 Pfoh ER, Wozniak AW, Colantuoni E, et al. Physical declines occurring after hospital discharge in ARDS survivors: a 5-year longitudinal study. Intensive Care Med 2016:42:1557-66.

49 Varadhan R, Weiss CO, Segal JB, et al. Evaluating health outcomes in the presence of competing risks: a review of statistical methods and clinical applications. Med Care 2010;48(6 Suppl):S96-105

50 Pandharipande PP, Girard TD, Jackson JC, et al. Long-term cognitive impairment after critical illness. N Engl J Med 2013;369:1306-16.

51 Maley JH, Brewster I, Mayoral I, et al. Resilience in survivors of critical illness in the context of the survivors' Experience and recovery. Ann Am Thorac Soc 2016;13:1351-60. 
52 Redekop WK, Mladsi D. The faces of personalized medicine: a framework for understanding its meaning and scope. Value Health 2013;16(6 Suppl):S4-S9.

53 President's Council of Advisors on Science and Technology. Priorities for Personalized Medicine. Washington, DC, 2008.

54 Herridge MS. The challenge of designing a post-critical illness rehabilitation intervention. Crit Care 2011;15:1002.
55 Hashem MD, Parker AM, Needham DM. Early mobilization and Rehabilitation of Patients who are critically III. Chest 2016;150:722-31.

56 Puthucheary ZA, Denehy L. Exercise interventions in critical illness survivors: understanding Inclusion and Stratification Criteria. Am J Respir Crit Care Med 2015;191:1464-7. 